\title{
Local burning velocity in a Bunsen jet flame
}

\author{
Gabriel García-Soriano a , José Luis Castillo ${ }^{\text {a }}$, Francisco J. Higuera ${ }^{\mathrm{b}}$, Pedro L. García-Ybarra ${ }^{\mathrm{a}, *}$ \\ a Dept. Fîsica Matemática y de Fluidos, UNED, Facultad de Ciencias, Senda del Rey 9, 28040 Madrid, Spain \\ ${ }^{\mathrm{b}}$ E.T.S. Ingenieros Aeronáuticos, UPM, Pza. Cardenal Cisneros, 28040 Madrid, Spain
}

\begin{abstract}
A B S T R A C T
A PIV-based system has been set-up for the simultaneous measurement of the local burning velocity of premixed flames and the flame stretch due to the flame front curvature and the incoming flow strain rate. For moderately short jet flames, these measurements allow an indirect determination of the Markstein length, according to Clavin and Joulin $(\mathrm{C}-\mathrm{J})$ theory. For tall flames, the flame curvature becomes relatively large in a region around the tip where the $\mathrm{C}-\mathrm{J}$ theory breaks down. However, our experiments confirm the appearance of a new linear relation between burning velocity and curvature at the flame tip. This relation defines a new proportionality factor which is probably associated to the evolution from rounded tips to slender tips when the jet velocity is increased.
\end{abstract}

\section{Introduction}

The pioneering ideas of Markstein [1], Ekhaus [2] and Chu and Parlange [3], among others, about the link between the local burning velocity of a premixed flame and its departure from the planar unstretched flame configuration crystallized during the 1980s in a series of works that settled this link on firm grounds; see for instance the review paper of Clavin [4] and the references therein. In the low Mach number limit, assuming that the gasdynamic variables vary over some spatial length $L$ (the flame curvature radius say) which is large compared to the flame thermal thickness $d$, the flame appears as a thin front separating two isothermal zones: the fresh gas and the burned gas. The thermal structure of the flame was analyzed in these conditions by assuming a quasi-planar configuration for the distorted front and exploiting the simplifications brought about by the limit $d / L \ll 1$ (see Williams [5]). One of the main results of this analysis was the finding that the relative departure of the local burning velocity $U_{n}$ from the planar flame velocity $U_{L}$ is proportional to the flame stretch; i.e. to the relative rate of change of area of the propagating flame due to its curvature and to the strain rate of the fresh gas flow immediately ahead of the flame. The burning velocity decreases below $U_{L}$ when the stretch is positive (expanding flame) and increases above $U_{L}$ when the stretch is negative (compressing flame) according to

$$
\frac{U_{n}}{U_{L}}-1=\mathcal{L}\left(\nabla \cdot \boldsymbol{n}+\frac{1}{U_{L}} \boldsymbol{n} \cdot \nabla \boldsymbol{v} \cdot \boldsymbol{n}\right)+O\left(\frac{d}{L}\right)^{2}
$$

irrespective of the amplitude of the front corrugation [6,7]. The term in parentheses on the right-hand side of this equation is the flame stretch. It is made of the two contributions mentioned above. The first contribution is due to the front curvature,

\footnotetext{
* Corresponding author.

E-mail address: pgybarra@dfmf.uned.es (P.L. García-Ybarra).
} 


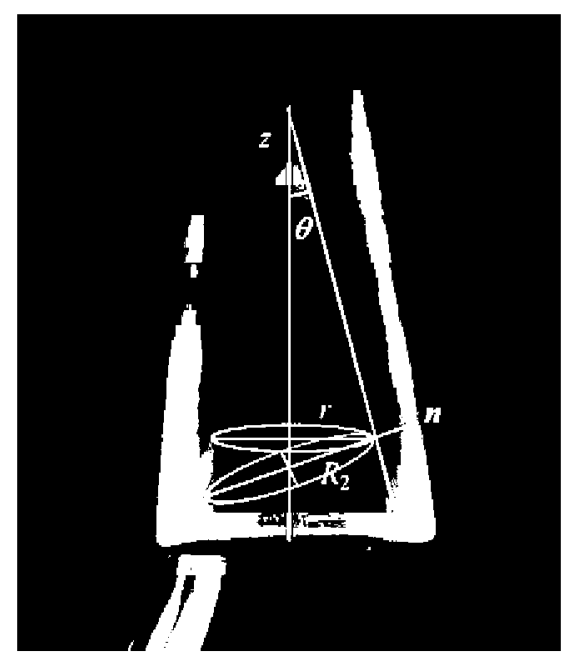

Fig. 1. Laminar jet flame in a rich methane-air mixture. It consists of a quasi-conical premixed flame surrounded by a diffusion flame.

with $\boldsymbol{n}$ the normal unit vector to the flame surface pointing towards the burnt gas, in such a way that its divergence is twice the mean flame curvature,

$$
\boldsymbol{\nabla} \cdot \boldsymbol{n}=\frac{1}{R_{1}}+\frac{1}{R_{2}}
$$

where $R_{1}$ and $R_{2}$ are the principal radii of curvature of the distorted front. The second contribution to the stretch is due to the rate of strain of the gas flow, $\boldsymbol{n} \cdot \nabla \boldsymbol{v} \cdot \boldsymbol{n}$, where $\boldsymbol{v}$ is the velocity of the fresh gas. The analysis provides also an expression of the proportionality constant, the Markstein length $\mathcal{L}$, which has been obtained under very general conditions in terms of the physicochemical properties of the reactive mixture [8-14]. It is worth noticing that this interpretation in terms of the stretch holds because Eq. (1) is a first order result in an asymptotic solution for $d / L \ll 1$ and the normal front velocity $U_{n}$ can be substituted by the planar flame velocity, $U_{n}=U_{L}[1+O(d / L)]$, in the right-hand side of $(1)$.

Eq. (1) will be termed the $\mathrm{C}-\mathrm{J}$ relation in what follows, a shorthand for Clavin and Joulin [7]. Despite the time elapsed since this relation was derived, little is known about its precise limits of validity and its possible extensions when flame fronts appear curved on small scales of the order of the flame thermal thickness, $d / L=O(1)$. In principle, a well established theoretical framework exists that could be used to compute higher order terms in an expansion in powers of $d / L$. In practice, this computation soon meets serious algebraic difficulties that have prevented significant advance and have led to explore alternative ways.

An experimental approach to the problem was reported in [15] where, as in previous works [16,17], a PIV system was used to determine the gas flow upstream of a jet flame with quasi-conical shape, and the validity of $\mathrm{C}-\mathrm{J}$ relation was tested for premixed flames in rich mixtures of methane-air. This work was complemented by asymptotic and numerical studies [18,19], and further improvements of the experimental setup which allow a better control of the flame tip position and reduce the noise intensity will be reported here.

In the next section, the $\mathrm{C}-\mathrm{J}$ relation is used to predict the shape of jet flames in laminar streams with flat velocity profiles. Section 3 describes the experimental facility where the flow field and the geometry of jet premixed flames of rich methane-air mixtures were investigated, while the techniques used to measure the burning velocity and curvature of the flame and the strain rate of the fresh gas flow are presented in Section 4. The experimental results are analyzed and discussed in Section 5. Finally the main conclusions of the study are presented in Section 6.

\section{Prediction of the flame shape by the Clavin-Joulin theory}

The Clavin-Joulin relation (1) can predict the shape of a premixed flame in different configurations when the thermal thickness of the flame is small compared to its curvature radii and to the inverse of the strain rate of the fresh gas velocity divided by $U_{L}$; see, e.g. [20]. We consider here the case of a jet of reactive gas issuing upward from a vertical nozzle of radius $R$ with uniform velocity. When this velocity is larger than the planar flame velocity and the flat flame is morphologically stable, ignition leads to a premixed flame which is conical everywhere except in a region around its apex where the flame surface forms a rounded cap, like in Fig. 1.

In this section, the vertical distance $(z)$ and the distance to the axis of the tube $(r)$ are scaled with the Markstein length of the flame, $\mathcal{L}$; the velocities are scaled with $U_{L}$; and the shape of the flame is sought in the form $z=z_{f}(r)$. In the cylindrical coordinates $(z, r)$, the unit vector normal to the flame (pointing toward the burnt gas) and the mean curvature of the flame are 


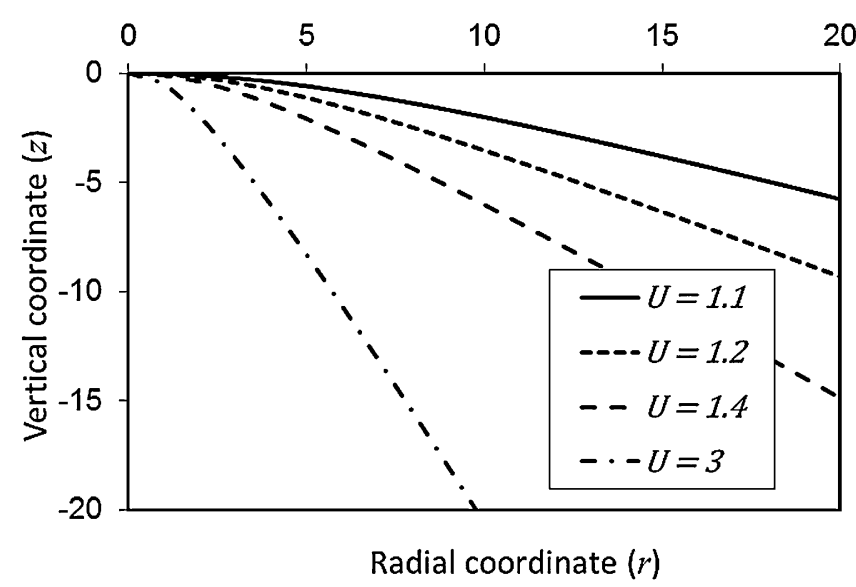

Fig. 2. Predicted shape of a jet flame for different values of the injection velocity.

$$
\boldsymbol{n}=\left(n_{z}, n_{r}\right)=\frac{\left(1,-z_{f}^{\prime}\right)}{\sqrt{1+z_{f}^{\prime 2}}} \text { and } \nabla \cdot \boldsymbol{n}=-\frac{1}{r}\left(\frac{r z_{f}^{\prime}}{\sqrt{1+z_{f}^{\prime 2}}}\right)^{\prime}
$$

where primes denote $r$-derivatives.

We consider first the case in which the dimensionless velocity of the gas is $U$, uniform. Then, since the normal velocity of a stationary flame coincides with the component of the fresh gas velocity normal to the flame, $U_{n}=U / \sqrt{1+z_{f}^{\prime 2}}$, Eq. (1) takes the form

$$
z_{f}^{\prime \prime}=\left[\sqrt{1+z_{f}^{\prime 2}}-\frac{z_{f}^{\prime}}{r}-U\right]\left(1+z_{f}^{\prime 2}\right)
$$

to be solved with the conditions

$$
z_{f}(0)=z_{f}^{\prime}(0)=0
$$

where the origin of $z$ is set at the tip of the flame and the second condition (4) is a condition of regularity at the symmetry axis.

The radius of the osculating sphere at the tip of the flame is, from (3), $\mathcal{R}=\left.2(\boldsymbol{\nabla} \cdot \boldsymbol{n})^{-1}\right|_{r=0}=2 /(U-1)$. Fig. 2 shows some results of the numerical integration of (3) and (4) for various values of the dimensionless fresh gas velocity $U$. It is worth noticing that Eq. (3) also predicts convex flames when $U<1$; i.e. a stationary flame may exist, without flashback, in a stream of gas with a velocity smaller than $U_{L}$.

Previous studies $[15,16,18]$ have shown that the presence of the flame modifies the velocity of the fresh gas by introducing an upward acceleration whose origin may be traced to the pressure variations in the non-uniform flow downstream of the flame. Experimental and numerical results show that the vertical component of the fresh gas velocity increases nearly linearly with vertical distance above the injection nozzle. The effect of this vertical acceleration on the shape of the flame can be easily analyzed replacing the uniform velocity assumed above by a linearly varying dimensionless velocity

$$
\boldsymbol{v}=\left(U_{\text {tip }}+A z,-\frac{1}{2} A r\right)
$$

where $U_{\text {tip }}$ is the velocity of the fresh gas at the flame tip, $A$ is a constant, and the radial velocity component $-\frac{1}{2} A r$ is computed from the continuity equation. The strain rate term in (1) evaluated with this velocity is $\boldsymbol{n} \cdot \nabla \boldsymbol{v} \cdot \boldsymbol{n}=$ $A\left(1-z_{f}^{\prime 2} / 2\right) /\left(1+z_{f}^{\prime 2}\right)$, which is positive, leading to an increase of the flame velocity, in a region around the tip where $\left|z_{f}^{\prime}\right|<\sqrt{2}$, and negative at the rest of the flame. The component of the fresh gas velocity normal to the flame is

$$
U_{n}=\boldsymbol{v} \cdot \boldsymbol{n}=\left[U_{\text {tip }}+A\left(z_{f}+r z_{f}^{\prime} / 2\right)\right] / \sqrt{1+z_{f}^{\prime 2}}
$$

and Eq. (1) takes the form

$$
z_{f}^{\prime \prime}=\left[\sqrt{1+z_{f}^{\prime 2}}-\frac{z_{f}^{\prime}}{r}+A\left(\frac{1-z_{f}^{\prime 2} / 2}{\sqrt{1+z_{f}^{\prime 2}}}-\frac{r z_{f}^{\prime}}{2}-z_{f}\right)-U_{\text {tip }}\right]\left(1+z_{f}^{\prime 2}\right)
$$

to be solved with conditions (4). 


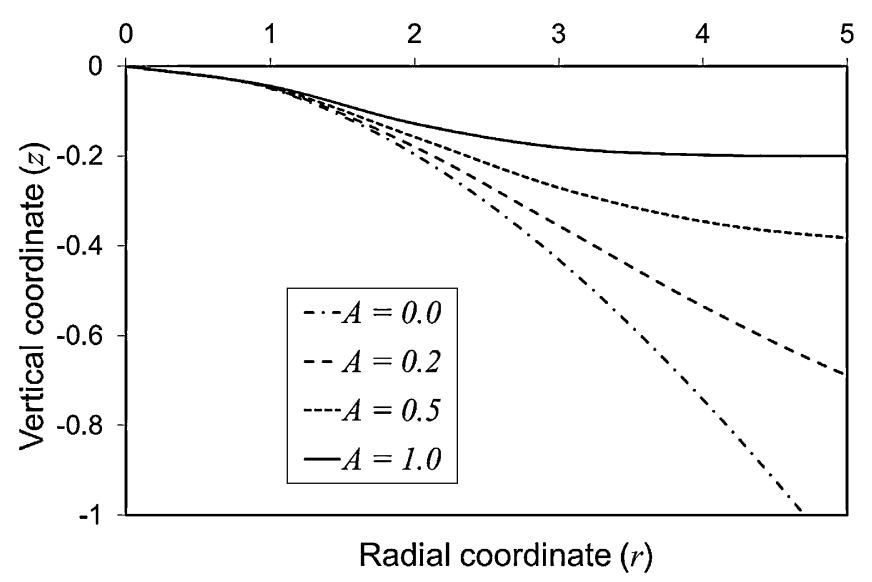

Fig. 3. Flame shapes in the presence of a vertical gradient of the fresh gas velocity, for $\mathcal{R}=10$.

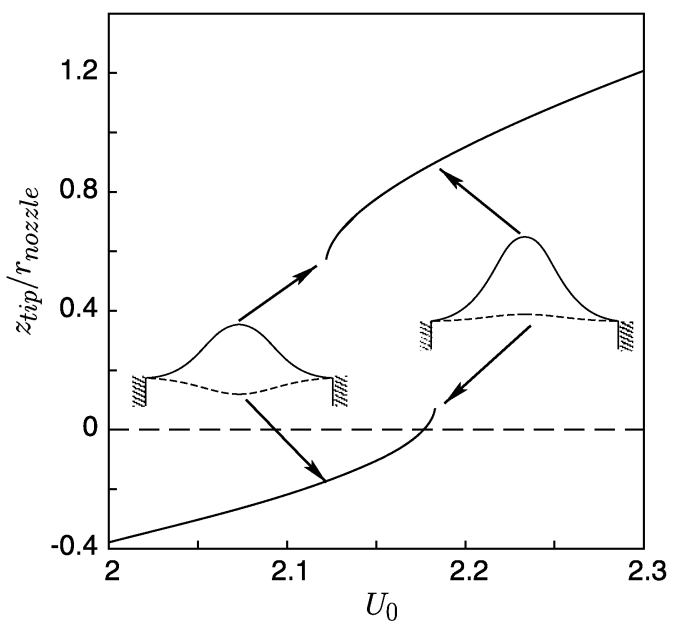

Fig. 4. Bistable behavior occurring in the presence of a vertical gradient of the fresh gas velocity.

The radius of the osculating sphere at the flame tip is now $\mathcal{R}=2 /\left(U_{\text {tip }}-1-A\right)$. Some numerical solutions of (6) and (4), which depend on the two dimensionless parameters $U_{\text {tip }}$ and $A$, are shown in Fig. 3 . These solutions have been computed for $\mathcal{R}=10$; i.e. $U_{\text {tip }}=1.2+A$.

The dimensionless velocity with which the fresh gas issues from the nozzle to which the flame is attached is $U_{0}=$ $U_{\text {tip }}-A z_{\text {tip }}$, where $z_{\text {tip }}=-z_{f}\left(r_{\text {nozzle }}\right)$ is the dimensionless height of the flame tip above the nozzle and $r_{\text {nozzle }}=R / \mathcal{L}$ is the dimensionless radius of the nozzle. The solution of (6) when the finite radius of the nozzle is taken into account depends on the three dimensionless parameters $\left(U_{\text {tip }}, A, r_{\text {nozzle }}\right)$ or, equivalently, $\left(U_{0}, A, r_{\text {nozzle }}\right)$. Fig. 4 shows the height of the flame above the nozzle computed as a function of $U_{0}$ for $A=1.17$ and $r_{\text {nozzle }}=3.33$. As can be seen, there is a range of bistability. A relatively tall flame with large $U_{\text {tip }}$ and small $\mathcal{R}$ (solid curves in the inserts of Fig. 4) exists for $U_{0}$ above a certain minimum $U_{01} \approx 2.122$, and a shorter flame with smaller $U_{\text {tip }}$ and larger $\mathcal{R}$ (dashed curves in the inserts of Fig. 4 ) exists below a certain maximum $U_{02} \approx 2.183>U_{01}$. If the linear velocity distribution (5) is assumed to extend to the interior of the nozzle, which is qualitatively correct for the convergent nozzle used in our experiments, then the curvature at the tip changes from concave to convex toward the fresh gas, and the flame gets into the nozzle, when $U_{0}$ decreases along the lower solution branch.

As was mentioned in the introduction, all these results are valid only for weak stretch; i.e. the departure of the dimensionless burning velocity from unity must by of order $d / L$. This condition is not satisfied when $U_{0}-1$ becomes of order unity or large, in which case the flame radius of curvature may become of the order of the flame thickness and the predictions of the $C-J$ theory cannot be expected to be accurate. The limit $U_{0} \gg 1$, leading to slender flames, was analyzed by Buckmaster [21] and Buckmaster and Crowley [22], and revisited in [18,19]. The work [19] examines the double asymptotic limit of large fresh gas velocity and large thermal expansion across the flame; $U_{0} \gg 1$ and $\Theta=T_{b} / T_{u} \gg 1$, where $T_{u}$ and $T_{b}$ are the initial temperature of the fresh gas and the adiabatic flame temperature, respectively. Various asymptotic structures of the flame tip, ranging from rounded to slender tips, have been described depending on the order of the ratio $U_{0} / \Theta$, and a transition between two qualitatively different solutions has been predicted for $U_{0}=O(\Theta) \gg 1$. 


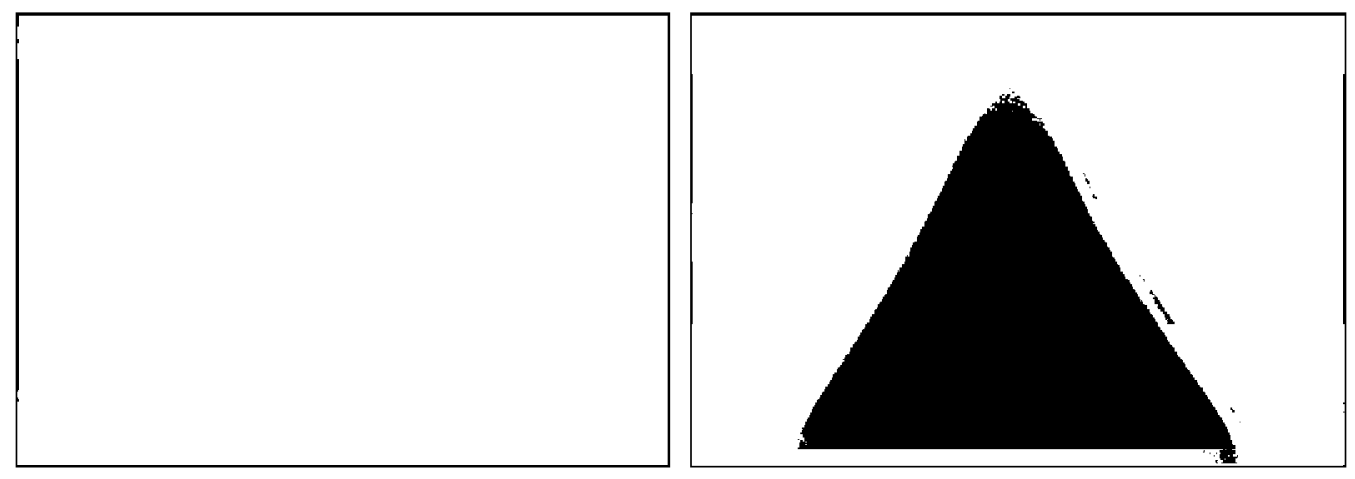

Fig. 5. Typical pair of pictures obtained with the PIV system. The pictures shown are actually the negative of the originals. The second picture (on the right) distinctly shows the luminescence from the reaction sheet as a halo surrounding the image of laser light reflected by the oil droplets.

\section{Experimental facility}

The apparatus used to produce a premixed jet flame is similar to that used in [15] and will be described only briefly. It consists of a vertical cylindrical tube $230 \mathrm{~mm}$ long which ends in a convergent nozzle with 4:1 contraction ratio and $10 \mathrm{~mm}$ final radius. The tube is fitted with various porous discs, grids and honeycombs to uniformize the flow and achieve a nearly uniform velocity profile at the end of the nozzle. The lower end of the tube is connected to a plenum chamber which is fed with a methane-air mixture of adjustable equivalence ratio. This mixture is prepared from separate streams of methane and air metered with Bronkhost mass flow controllers. The original apparatus described in [15] has been modified in two ways.

- The wall of the tube has been thermalized with a water jacket kept at a constant temperature by means of a heat exchanger. This prevents the uncontrolled heating of the reacting gas by the tube wall which was observed in our previous experiments.

- A controlled air coflow has been added around the nozzle to reduce flickering and help stabilize the tip of the flame.

Flow velocity measurements are made with a PIV optical diagnostic system composed of a pulsed Nd:YAG laser, a lasersheet forming emission optics, a high speed shuttering CCD camera (1,392 $\times 1,040$ pixels) and a pulse-delay generator (see [15] for details).

Oil droplets are used as flow tracers in the cold gas upstream of the flame. The droplets are generated in a chamber inserted in the air line by condensation following evaporation over a hot plate at $450^{\circ} \mathrm{C}$. They are carried by the air stream into the plenum chamber, which acts as a settling chamber to eliminate the largest droplets. The remaining droplets are entrained by the reactive mixture and are further filtered in their passage through the various bodies inside the tube, so that the drops emerge from the nozzle with a fairly narrow size distribution.

\section{Flame tomography and mean curvature}

The PIV system generates pairs of pictures of a meridian section of the fresh gas flow ahead of the flame; a typical pair is shown in Fig. 5. The time between laser pulses (interframing time) was $70 \mu \mathrm{s}$. The exposure time of the first picture was about $500 \mu \mathrm{s}$, while the exposure time of the second picture cannot be adjusted; it is determined by the readout time of the camera, which is comparatively large, about $100 \mathrm{~ms}$. This time is long enough for the radiation emitted by the flame to form an image on the second picture. The outline of the luminous region of the flame is separated from the light scattered by the oil droplets by a dark fringe which corresponds to the flame preheating region, because the droplets rapidly evaporate on entering this region. This fact allows to build a tomography of the flame which can be superimposed onto the velocity field obtained from the PIV correlation analysis.

An edge detection algorithm based on a Laplacian-of-Gaussian filter has been developed to extract the location of the reaction sheet from the second picture of each pair. This method proved to be more convenient than alternative methods based on the Abel transform, which have been often used for problems involving axisymmetric radiation. In fact, Abel transform methods do not account for perspective and out-of-focus effects. However, in the present experiments, with a depth of field less than $1 \mathrm{~mm}$ and an image depth of tens of millimeters, most of the image is out of focus and such effects cannot be neglected. Fig. 6 shows a sample of reaction sheet and droplet depletion contours extracted with our method and superimposed on the original picture.

A fifth order spline has been used to fit the reaction sheet and compute its curvature. The maximum departure of the spline curve from the detected reaction sheet was set to 1.2 pixels, an accuracy that was achieved using about five knots on the whole tip region. 


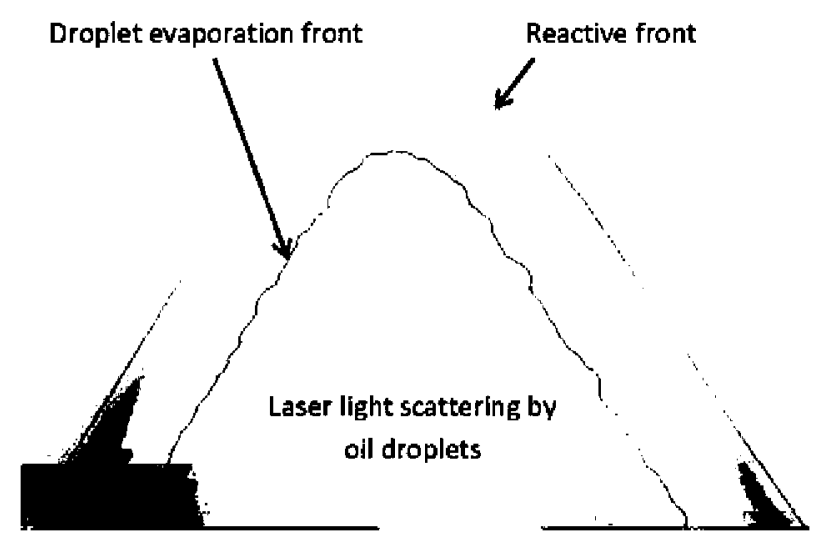

Fig. 6. Location of the reaction sheet and the droplet depletion front obtained by the edge detection algorithm for a sample flame picture.

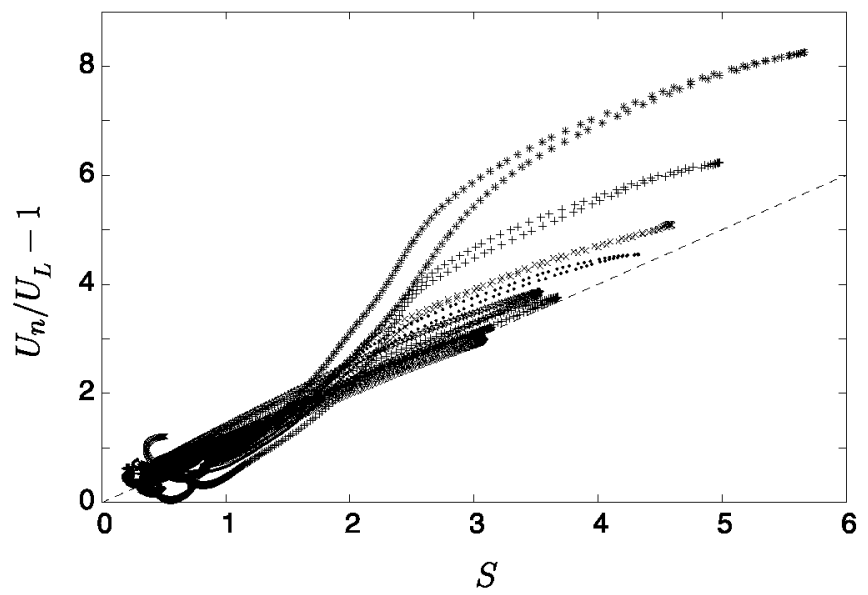

Fig. 7. Local excess of dimensionless burning velocity as a function of local dimensionless stretch along flames of various heights. The dashed line has unit slope and corresponds to the $\mathrm{C}-\mathrm{J}$ relation in the present dimensionless variables. Short flames follow this relation everywhere. Tall flames depart from the $\mathrm{C}-\mathrm{J}$ relation in a region around their tip.

The normal burning velocity $U_{n}$ and the curvature and strain rate contributions to the stretch need to be evaluated to experimentally assess the $\mathrm{C}-\mathrm{J}$ relation. The normal velocity appearing in (1) is the velocity of the fresh gas upstream of the flame extrapolated to the reaction sheet. This theoretical construct has a fairly direct experimental translation. Since the boundary of the oil droplet region sharply marks the beginning of the flame preheating region, the velocity field measured with the PIV system is that of the cold gas, and extrapolation to any point of the reaction sheet is straightforward. Moreover, as was advanced in Section 2, the measured velocity has a nearly uniform vertical gradient $(A)$ in the whole region occupied by the droplets, and the radial velocity associated to this vertical gradient is typically less than $5 \%$ of the vertical velocity and makes a negligible contribution to $U_{n}$. The mean curvature of the reaction sheet is evaluated using the spline fitting mentioned above. The strain rate is $\boldsymbol{n} \cdot \nabla \boldsymbol{v} \cdot \boldsymbol{n}=A\left(n_{z}^{2}-n_{r}^{2} / 2\right)$ in terms of the vertical velocity gradient $A$ and the unit normal to the reaction sheet $\boldsymbol{n}=\left(n_{z}, n_{r}\right)$, both of which are available from the experimental data. The contribution of the curvature to flame stretch is typically large compared to the contribution of the strain rate.

\section{Results and discussion}

The planar flame velocity $U_{L}$ and the Markstein length $\mathcal{L}$ are determined by plotting the burning velocity versus the flame stretch and using a linear regression for points with small stretch. This process requires iteration because $U_{L}$ appears in the expression of the stretch; see Eq. (1).

Fig. 7 shows results for eight methane-air flames with $\Phi=1.48$ and different values of the injection velocity, leading to different heights of the flames. Each curve gives $U_{n} / U_{L}-1$ as a function of $S=\mathcal{L}\left(\nabla \cdot \boldsymbol{n}+U_{L}^{-1} \boldsymbol{n} \cdot \nabla \boldsymbol{v} \cdot \boldsymbol{n}\right)$ along the reaction sheet of a flame, with the rightmost point of the curve corresponding to the tip of the flame. The values of the planar flame velocity and the Markstein length are $U_{L}=102 \mathrm{~mm} / \mathrm{s}$ and $\mathcal{L}=3.1 \mathrm{~mm}$, in reasonable accord with the results available in the literature for this equivalence ratio [23-26]. The dashed line in Fig. 7 shows the $C-J$ prediction. As can be seen, all 


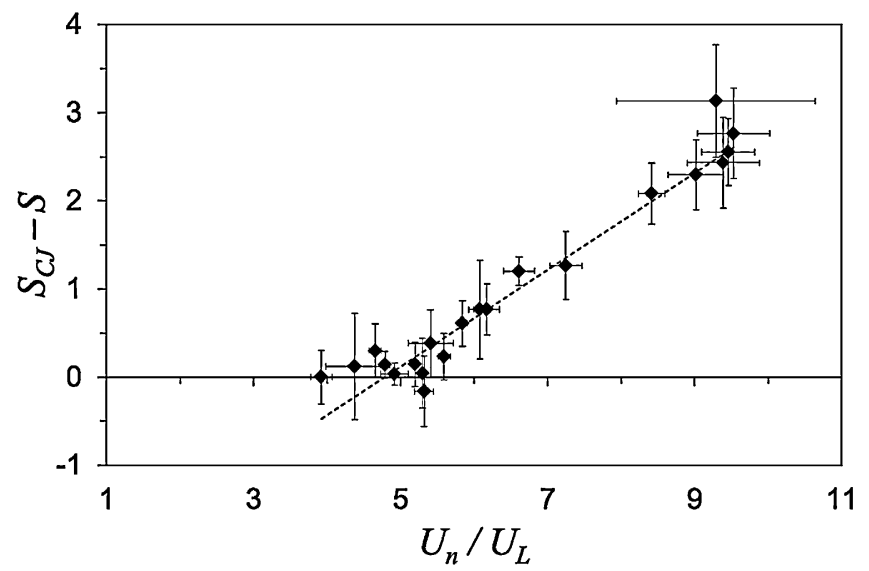

Fig. 8. Defect of stretch with respect to the $C-J$ prediction $\left(S_{C J}\right)$ at the tips of various flames versus the gas velocity at the tip. Each point is an average typically over more than ten measurements made under the same conditions. The error bars give the standard deviation. The dashed line is the linear correlation $S_{C J}-S=0.55\left[U_{n} / U_{L}-\left(U_{n} / U_{L}\right)_{0}\right]$, with $\left(U_{n} / U_{L}\right)_{0}=4.77$.

the experimental curves approach this line away from the tip, where the stretch is small, but the results for tall flames noticeably depart from the C-J prediction in a region around the flame tip where the flame stretch (due mostly to the curvature of the flame) is large.

Fig. 7 displays several remarkable facts, apart from the collapse of the data for all the flames onto the C-J prediction in regions of weak stretch. The shortest flames closely follow the linear C-J relation all along their reaction sheet, as could be expected. More inclined flames with larger curvature at the tip, obtained by increasing the injection velocity, still follow the C-J relation even though the burning velocity at the tip may reach values of about five times $U_{L}$. A transition is observed when the injection velocity is further increased. The normal velocity of the tallest flames in Fig. 7 increases above the C-J prediction in a region around their tip which extends nearly to the point where the strain rate changes sign. Curvature and strain seem to reinforce each other in this region, leading to an increase of the flame velocity higher than the sum of their separate contributions. The difference with the C-J prediction increases as the tip is approached, until a saturation level is attained where the velocity/stretch relation becomes again linear with a slope similar to that of C-J but with an upward shift of the flame velocity.

As a tentative explanation of this result, pending closer scrutiny, we may note that the new linear relation is compatible with the C-J theory if the initial temperature of the fresh gas that burns around the flame tip is assumed to be slightly higher than the temperature with which this gas leaves the injection nozzle, which would lead to an increase of the velocity of a planar flame propagating in this gas. For tall flames, such a temperature increase may be brought about by the small but cumulative heating experienced by the fresh gas that flows nearly parallel to the flame before actually entering its preheating region. The effect increases with the inclination of the flame [19] and, for a given flame, it is most pronounced for the gas flowing near the symmetry axis, in line with the results in Fig. 7.

It may also be said that the one-to-one relation between burning velocity and stretch characteristic of the C-J theory breaks down due to the increase of initial gas temperature. Since this temperature increase changes from flame to flame, the burning velocity for a given stretch (on the horizontal axis of Fig. 7) is different for different flames.

The magnitude of the velocity shift above the C-J prediction increases rather abruptly with the injection velocity. This result, which was already found in [15] from a comparison of experimental and numerical data, permits to define a threshold injection velocity below which the C-J relation holds at the flame tip and above which the tip experiences a lowered stretch (e.g., the tip is less curved than the C-J theory predicts for its velocity). This transition is depicted in Fig. 8 and implies a reduction in the tendency toward the formation of sharp flame tips. The critical value of the gas velocity is seen to be close to $5 U_{L}$.

The analysis of [19] for very tall and exothermic flames, $\left(U_{0}, \Theta\right) \rightarrow \infty$, predicts a transition in the structure of the flame tip from round to slender when the value $U_{n} / U_{L}$ at the tip becomes of the order of the temperature ratio $\Theta=T_{b} / T_{u}$. This analysis may also be tickled to produce a very rough estimate of the temperature increase experienced by the fresh gas that approaches the tip of the flame as $\Delta T /\left(T_{b}-T_{u}\right)=O\left(\Theta^{-1}\right)$. In our experiments, using the values $T_{b} \approx 1950 \mathrm{~K}$ and $T_{u} \approx 300 \mathrm{~K}$, the temperature ratio is $\Theta \approx 6.5$ and $\Delta T \sim 250 \mathrm{~K}$. The first result shows that the theoretical estimation of $U_{n}$ at transition is of the order of the critical velocity in Fig. 8. As for the second result, we note that, according to classical flame theory [5], a fresh gas temperature increment $\Delta T$ would cause an increase of the planar velocity from $U_{L}$ to $\widetilde{U}_{L}=U_{L} \exp \left(\beta \Delta T / 2 T_{b}\right) \sim 2.6 U_{L}$, where $\beta=E_{a} / R T_{b} \approx 15$ is an effective dimensionless activation energy. This $\widetilde{U}_{L}$ should play the role of $U_{L}$ when the data in Fig. 7 for points around the tips of tall flames are extrapolated to zero stretch as explained above, which is qualitatively correct. These comparisons support the view that the observed change in the behavior of the flame tips corresponds to the theoretically expected transition from round tips to slender tips. 
The dependence of the critical value of the gas injection velocity on the equivalence ratio of the mixture will be investigated in a follow-on work where the set-up will be modified to stabilize flame tips of lean mixtures. This modification should allow us to observe flames with open tips, as those analyzed by Sivashinsky [27].

\section{Conclusions}

An experimental methodology based on PIV, which was established in [15], has been developed further and used here to simultaneously measure the local burning velocity of premixed jet flames and the flame stretch due to the flame front curvature and the strain rate of the incoming flow.

The velocity-to-stretch ratio measured in regions of weak stretch along meridional sections of axisymmetric flames determines the Markstein length of the flame, in accordance with Clavin-Joulin theory.

The Clavin-Joulin theory is found to be valid all along the flame when the gas injection velocity is below a certain threshold, even though the stretch and the stretch-induced change of the burning velocity relative to the planar flame velocity are not small around the tip of the flame.

For values of the gas injection velocity above this threshold, the curvature of the flame is smaller than predicted by the Clavin-Joulin theory in a certain region around the flame tip or, what amounts to the same, the burning velocity in this region is larger than expected for the local curvature of the flame. A tentative explanation of this result is put forward in terms of the transition from round to slender tips predicted in [19].

\section{Acknowledgements}

This work was supported by the Comunidad de Madrid (Spain) through Project HYSYCOMB, S2009/ENE-1597 and by the Ministerio de Economía y Competitividad Program ConsoliderIngenio 2010 (CSD2010-00011) and project ENE2011-26868.

\section{References}

[1] G.H. Markstein, Non Steady Flame Propagation, Pergamon Press, 1964.

[2] W. Eckhaus, Theory of flame-front stability, J. Fluid Mech. 10 (1961) 80-100.

[3] B.T. Chu, J.Y. Parlange, On the stability of laminar flame, J. Mécanique 1 (1962) 293-312.

[4] P. Clavin, Premixed combustion and gasdynamics, Annu. Rev. Fluid Mech. 26 (1994) 321-352.

[5] F.A. Williams, Combustion Theory, 2nd ed., Benjamin Cummings, 1985.

[6] M. Matalon, B.J. Matkowsky, Flames as gasdynamic discontinuities, J. Fluid Mech. 124 (1982) 239-259.

[7] P. Clavin, G. Joulin, Premixed flames in large scale and high intensity turbulent flow, J. Phys. Lett. 44 (1983) L-1-L-12.

[8] P. Clavin, F.A. Williams, Effects of molecular diffusion and of thermal expansion on the structure and dynamics of premixed flames in turbulent flows of large scale and low intensity, J. Fluid Mech. 116 (1982) 251-282.

[9] P. Pelcé, P. Clavin, Influence of hydrodynamics and diffusion upon the stability limits of laminar premixed flames, J. Fluid Mech. 124 (1982) $219-237$.

[10] M.L. Frankel, G.I. Sivashinsky, The effect of viscosity on hydrodynamic stability of a plane flame front, Combust. Sci. Technol. 29 (1982) $207-224$.

[11] P. Clavin, P. Garcia, The influence of the temperature dependence of diffusivities on the dynamics of flame fronts, J. Mec. Theor. Appl. 2 (1983) 245-263.

[12] P. Garcia-Ybarra, C. Nicoli, P. Clavin, Soret and dilution effects on premixed flames, Combust. Sci. Technol. 42 (1984) 87-109.

[13] C. Nicoli, P. Clavin, Effect of variable heat loss intensities on the dynamics of a premixed flame front, Combust. Flame 68 (1987) 69-71.

[14] P. Clavin, J.C. Graña-Otero, Curved and stretched flames: the two Markstein numbers, J. Fluid Mech. 686 (2011) $187-217$.

[15] G. Garcia-Soriano, P.L. Garcia-Ybarra, F.J. Higuera, Curvature and velocity of methane-air Bunsen flame tips, Flow Turbul. Combust. 89 (2012) 173-182.

[16] T. Echekki, M.G. Mungal, Flame speed measurements at the tip of a slot burner: Effects of flame curvature and hydrodynamic stretch, Proc. Combust. Inst. 23 (1990) 455-461.

[17] M.G. Mungal, L.M. Lourenco, A. Krothapalli, Instantaneous velocity measurements in laminar and turbulent premixed flames using on-line PIV, Combust. Sci. Technol. 106 (1995) 239-265.

[18] F.J. Higuera, Aerodynamics of a slender axisymmetric Bunsen flame with large gas expansion, Combust. Flame 156 (2009) $1063-1067$.

[19] F.J. Higuera, Effects of fresh gas velocity and thermal expansion on the structure of a Bunsen flame tip, Combust. Flame 157 (2010) $1586-1593$.

[20] M. Matalon, On flame stretch, Combust. Sci. Technol. 31 (1983) 169-181.

[21] J. Buckmaster, A mathematical description of open and closed flame tips, Combust. Sci. Technol. 20 (1979) 33-40.

[22] J. Buckmaster, A.B. Crowley, The fluid mechanics of flame tips, J. Fluid Mech. 131 (1983) 341-361.

[23] G. Searby, J. Quinard, Direct and indirect measurements of Markstein numbers of premixed flames, Combust. Flame 82 (1990) $298-311$.

[24] B. Deshaies, P. Cambray, The velocity of a premixed flame as a function of the flame stretch: an experimental study, Combust. Flame 82 (1990) $361-375$.

[25] S.G. Davis, J. Quinard, G. Searby, Determination of Markstein numbers in counterflow premixed flames, Combust. Flame 130 (2002) $112-122$.

[26] S.G. Davis, J. Quinard, G. Searby, Markstein numbers in counterflow, methane- and propane-air flames: a computational study, Combust. Flame 130 (2002) 123-136.

[27] G.I. Sivashinsky, The structure of Bunsen flames, J. Chem. Phys. 62 (1975) 638-643. 